Leila Posenato Garcia ${ }^{1}$ Lúcia Rolim Santana ${ }^{2}$
${ }^{1}$ Diretoria de Estudos e Políticas Sociais - Disoc, Instituto de Pesquisa Econômica Aplicada - Ipea. SBS Quadra 1, Bloco J, Ed. Ipea/BNDES, sala 1431. 70.076-900 Brasília DF. leila.garcia@ipea.gov.br ${ }^{2}$ Diretoria de Estudos e Políticas Setoriais de Inovação, Regulação e Infraestrutura - Diset, Instituto de Pesquisa Econômica Aplicada, Ipea

\section{Evolução das desigualdades socioeconômicas na mortalidade infantil no Brasil, 1993-2008}

\author{
Evolution of socioeconomic inequalities \\ in infant and child mortality in Brazil, 1993-2008
}

Abstract In Brazil, infant and child mortality reveal a downward trend. Few studies have investigated the temporal trends in the inequalities of these deaths, at individual level, according to socio-economic status. The scope of this article was to investigate the temporal trends of the magnitude of inequalities in infant and child mortality, between groups defined by maternal education and household income per capita in the 1993-2008 period in Brazil. Microdata from the National Household Sample Survey were used. The inequality measure was the concentration index (CI). It was calculated for infant and child deaths, according to maternal education and household income per capita. During the 2003-2008 period, there was a reduction in inequalities in infant and child mortality, at individual level, and according to maternal education and household income per capita. In 2008, the concentration of child deaths among children whose mothers had less education was higher than the concentration of infant deaths. The CI according to income had greater variability, but also demonstrated a lower concentration of deaths at the end of the period. The measurement of inequalities in health is important for monitoring the population health situation.

Key words Health inequalities, Infant mortality, Child mortality, Educational status, Income, Socio-economic survey
Resumo No Brasil, a mortalidade infantil e na infância apresentam tendência decrescente. Poucos estudos investigaram a evolução temporal das desigualdades socioeconômicas nesses óbitos, no nivel individual. O objetivo foi investigar a evolução temporal da magnitude das desigualdades na mortalidade infantil e na infância, segundo a escolaridade materna e a renda domiciliar per capita, no período 1993-2008, no Brasil. Foram utilizados microdados das Pesquisas Nacionais por Amostra de Domicílios (PNAD). A medida de desigualdade empregada foi o indice de concentração (IC), calculado para os óbitos infantis e na infância, segundo escolaridade materna e renda domiciliar per capita. No período de 1993 a 2008, houve redução das desigualdades na mortalidade infantil e na infância, no nivel individual, segundo a escolaridade materna e a renda domiciliar per capita. Em 2008, a concentração dos óbitos na infância entre crianças cujas mães tinham menor escolaridade era maior do que a concentração dos óbitos infantis. Os IC segundo a renda apresentaram maior variabilidade, mas também demonstraram menor concentração dos óbitos ao final do periodo. A medição das desigualdades em saúde é importante para o acompanhamento da situação de saúde da população.

Palavras-chave Desigualdades em saúde, Mortalidade infantil, Mortalidade na infância, Escolaridade, Renda 


\section{Introdução}

As taxas de mortalidade infantil (de menores de um ano) e na infância (de menores de cinco anos) são indicadores muito importantes, uma vez que têm um peso importante na expectativa de vida ao nascer. Esses indicadores têm sido historicamente utilizados para avaliar as condições de saúde e de vida de populações. Os óbitos de crianças são eventos indesejáveis, pois são mortes precoces e, na maioria dos casos, evitáveis. A redução da mortalidade infantil e na infância faz parte dos Objetivos de Desenvolvimento do Milênio (ODM), compromisso dos países membros da Organização das Nações Unidas (ONU) para que, com a globalização, o mundo se torne mais inclusivo e equitativo no novo milênio ${ }^{1}$.

É amplamente conhecida a associação direta entre condição socioeconômica e situação de saúde. Em nível individual, os mais ricos têm saúde melhor. Além disso, diversos autores sugerem que as desigualdades na sociedade como um todo contribuem para que a situação de saúde de indivíduos que vivem em vizinhanças pobres seja ruim $^{2}$. A redução das desigualdades em saúde e, em especial, na sobrevivência infantil, é atualmente alvo da atenção de organizações internacionais e do governo brasileiro. Uma dessas iniciativas é o Pacto pela Redução da Mortalidade Infantil na Região Nordeste e Amazônia Legal, que faz parte de um compromisso mais amplo, proposto pelo governo federal, para acelerar a redução das desigualdades regionais.

No Brasil, a taxa de mortalidade infantil apresenta tendência decrescente: em 1980 era 78,5 óbitos por mil nascidos vivos ${ }^{3}$ e, em 2008, era estimada em 19,1 óbitos por mil nascidos vivos. Contudo, ainda era muito superior àquela de diversos países. Por exemplo, em 2008, a taxa de mortalidade infantil em Portugal, na Suécia, no Japão, na França e na Itália era em torno de três óbitos por mil nascidos vivos. Cuba, Canadá, Espanha e Reino Unido apresentavam taxas entre quatro e cinco óbitos por mil nascidos vivos. Outros países da América Latina também apresentavam taxas inferiores ao Brasil, como Chile, Costa Rica, Argentina e Uruguai, que apresentavam, respectivamente, taxas iguais a sete, dez, 11 e 14 óbitos por mil nascidos vivos ${ }^{4}$. A taxa de mortalidade na infância também apresentou declínio. No Brasil, essa taxa era 53,7 e 22,8 óbitos por mil nascidos vivos, em 1990 e 2008, respectivamente.

Para atingir a meta dos ODM, o Brasil deverá apresentar uma taxa de mortalidade infantil inferior a 15,7 óbitos por mil nascidos vivos e uma mortalidade na infância inferior a 17,9 óbitos por mil nascidos vivos em 2015. Isso equivale a uma redução de dois terços em relação às taxas de $1990^{1}$.

Em 2007, as taxas de mortalidade infantil e na infância das regiões Sul (12,9 e 15,0 óbitos por mil nascidos vivos, respectivamente) e Sudeste (14,6 e 16,5 óbitos por mil nascidos vivos, respectivamente) já eram inferiores à meta estipulada, refletindo as desigualdades regionais existentes no país ${ }^{1}$.

Para mensurar a magnitude das desigualdades na mortalidade infantil, no nível individual, são necessários dados de inquéritos domiciliares que agreguem informações sobre óbitos infantis e nível socioeconômico. As Pesquisas Nacionais por Amostra de Domicílios (PNAD), realizadas pelo Instituto Brasileiro de Geografia e Estatística (IBGE), possibilitam esse tipo de análise.

Os objetivos do presente artigo são investigar a evolução temporal da magnitude das desigualdades na mortalidade infantil e na infância, segundo a escolaridade materna e a renda domiciliar per capita, no período de 1993 a 2008, no Brasil.

\section{Métodos}

Foram utilizados microdados das PNAD realizadas no período de 1993 a 2008. Nos anos de 1994 e 2000, não foi realizada PNAD.

Os desfechos de interesse foram os óbitos infantis (de menores de um ano) e na infância (de menores de cinco anos). Os desfechos foram definidos de maneira direta a partir das questões referentes às características de fecundidade, aplicadas às mulheres com dez ou mais anos de idade, que permitiam identificar o momento do nascimento dos filhos nascidos vivos e se o último filho nascido vivo ainda estava vivo na data de referência da entrevista estipulada em cada PNAD. A partir dessas informações, foram identificados os óbitos dos últimos nascidos vivos, menores de um e de cinco anos, filhos das mulheres entrevistadas.

As variáveis independentes analisadas foram a escolaridade materna, em anos de estudo, e a renda domiciliar mensal per capita, na moeda corrente, definida pelo IBGE. A evolução temporal das desigualdades na mortalidade infantil e na infância, segundo essas variáveis, foi avaliada por meio do índice de concentração (IC). O IC é uma medida de desproporcionalidade ponderada pelo tamanho populacional, que avalia o desequilíbrio entre a distribuição do desfecho entre 
grupos e a distribuição da população entre estes mesmos grupos. Na presente análise, o IC permite avaliar se a distribuição dos óbitos infantis e na infância segundo grupos de escolaridade ou renda é proporcional à distribuição dos nascimentos segundo estes grupos. Ele pode ser entendido como uma medida que sintetiza a desigualdade em dado aspecto de saúde de determinada população.

O IC é derivado de uma curva de concentração (Figura 1), que traça a proporção acumulada da variável de saúde (nesse caso, os óbitos infantis ou na infância) contra a proporção acumulada da população ordenada segundo a posição socioeconômica (nesse caso, segundo a escolaridade materna ou a renda domiciliar per capita) $)^{5,6}$.

O IC varia entre $-1 \mathrm{e}+1$. Na presente análise, o sinal negativo do IC indica maior concentração dos óbitos infantis ou na infância entre crianças cujas mães tinham menor escolaridade ou menor renda domiciliar per capita (desigualdade favorável à população com melhor condição socioeconômica). Por outro lado, o sinal positivo indica maior concentração dos óbitos infantis ou na infância entre crianças cujas mães tinham maior escolaridade ou maior renda domiciliar per capita (desigualdade favorável à população com pior condição socioeconômica), conforme se pode observar na Figura 1.

O sinal do IC indica a direção da possível relação existente entre a variável de saúde e a posição na escala socioeconômica, enquanto sua magnitude reflete a força da associação e o grau de variabilidade do desfecho na população ${ }^{6}$. Quanto mais próximo a zero, mais próximo à igualdade e quanto mais próximo a -1 ou +1 , maior a desigualdade.

Utilizando o método de aproximação linear, o IC pode ser estimado por meio da fórmula a seguir ${ }^{6,7}$ :

$\mathrm{IC}=2^{*} \Sigma\left[\mathrm{f}_{\mathrm{t}}^{*} \mu_{\mathrm{t}}^{*} \mathrm{R}_{\mathrm{t}}\right] / \mu-1$

Onde: $\mathrm{f}_{\mathrm{t}}$ é a parcela da população do grupo socioeconômico tà qual o indivíduo pertence; $\mu_{\mathrm{t}}$ é a média do grupo; $\mathrm{R}_{\mathrm{t}}=$ é a posição fracional na ordenação socieconômica e $\mu$ é média geral. Os erros-padrão, para o cálculo dos intervalos de confiança podem ser obtidos por meio do método da covariância ${ }^{7}$.

Atente-se que o IC depende da distribuição da variável independente (nesse caso, a escolaridade materna ou a renda domiciliar per capita) apenas para o ordenamento da população, não sendo afetado por ela, mas sim pela covariância entre a posição no ordenamento socioeconômico e a ocorrência da doença.
As análises foram realizadas com o software Stata, versão 10 , tomando em conta o peso da pessoa (inverso da fração amostral), determinado pela variável incluída pelo IBGE no conjunto de dados de cada PNAD. Esta variável foi utilizada na implementação da fórmula proposta por Kakwani et al. ${ }^{7}$ para o IC e seu erro-padrão.

\section{Resultados}

Os números de domicílios pesquisados, bem como outras informações relevantes, referentes às amostras das PNAD das quais foram extraídos os dados empregados no presente estudo, estão descritos na Tabela 1.

Embora, o número de domicílios pesquisados, o número de mulheres pesquisadas e o número de nascimentos que foram captados pelas PNAD tenham crescido ao longo do tempo, em 2008 o número de óbitos de menores de um e de cinco anos captados foi consideravelmente reduzido.

A Figura 2 mostra a evolução do IC dos óbitos infantis e na infância, segundo a escolaridade materna, no período de 1993 a 2008.

Para as desigualdades na mortalidade infantil conforme a escolaridade materna, em 1993, o IC foi -0,26957 (IC95\%: -0,35046; -0,18868), indicando forte concentração dos óbitos de menores de um ano entre as crianças cujas mães tinham baixa escolaridade. Em 2008, o IC foi -

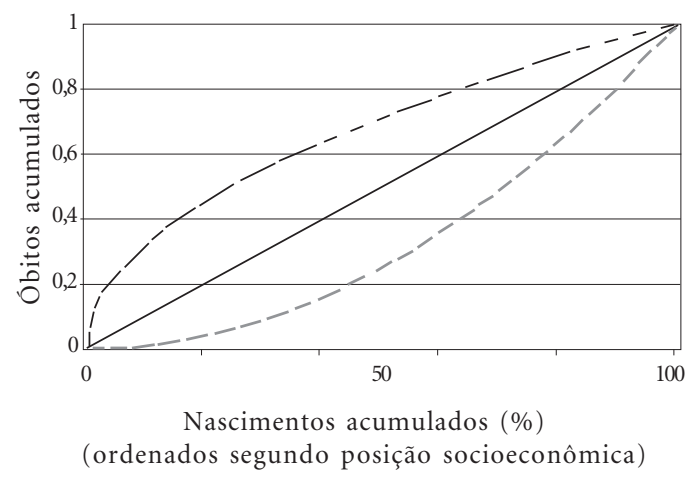

Linha de igualdade perfeita Desigualdade favorável a população com pior condição socio-econômica

- _ Desigualdade favorável a população com melhor condição socio-econômica

Figura 1. Curvas de concentração hipotéticas para óbitos infantis e na infância. 
Tabela 1. Distribuição da amostra analisada da PNAD no período de 1993 a $2008^{*}$

\begin{tabular}{|c|c|c|c|c|c|c|c|}
\hline & 1993 & 1995 & 1996 & 1997 & 1998 & 1999 & 2001 \\
\hline No de domicílios & 80.167 & 85.270 & 84.947 & 89.939 & 90.913 & 93.959 & 103.483 \\
\hline No de mulheres $\geq 10$ anos & 107.098 & 114.811 & 114.731 & 121.236 & 121.611 & 125.711 & 155.465 \\
\hline No de nascimentos $^{* *}$ & 74.447 & 79.732 & 79.464 & 84.501 & 85.002 & 87.732 & 96.624 \\
\hline No de óbitos $<1$ ano & 155 & 146 & 136 & 122 & 119 & 106 & 92 \\
\hline \multirow[t]{2}{*}{ No de óbitos $<5$ anos } & 592 & 521 & 392 & 477 & 438 & 427 & 397 \\
\hline & 2002 & 2003 & 2004 & 2005 & 2006 & 2007 & 2008 \\
\hline No de domicílios & 105.984 & 107.846 & 112.715 & 116.452 & 118.590 & 117.858 & 150.591 \\
\hline No de mulheres $\geq 10$ anos & 159.348 & 159.140 & 165.719 & 171.086 & 173.014 & 168.342 & 165.496 \\
\hline $\mathrm{No}^{\circ}$ de nascimentos ${ }^{* *}$ & 99.973 & 99.528 & 104.073 & 107.541 & 109.074 & 106.275 & 104.672 \\
\hline No de óbitos $<1$ ano & 76 & 107 & 92 & 86 & 84 & 80 & 52 \\
\hline No de óbitos $<5$ anos & 376 & 379 & 329 & 324 & 285 & 315 & 231 \\
\hline
\end{tabular}

*Elaboração dos autores, a partir dos microdados das Pesquisas Nacionais por Amostra de Domicílios (PNAD) 1993 a 2008. Nos anos de 1994 e 2000 não foi realizada PNAD. ${ }^{* *}$ Número de mulheres de 10 anos e mais que referiram ter tido algum filho nascido vivo até a data de referência (período de um ano anterior à realização da pesquisa).

0,06790 (IC95\%: -0,20330; 0,06750;), próximo de zero, indicando uma distribuição mais homogênea dos óbitos infantis segundo o número de anos de estudo da mãe. Entretanto, os intervalos de confiança de $95 \%$ se sobrepõem, demonstrando ausência de diferença estatisticamente significativa entre os valores do IC no início e no final do período.

Da mesma maneira como observado para a mortalidade infantil, o IC da mortalidade na infância segundo a escolaridade materna teve um valor negativo mais distante de zero em 1993 (0,33691; IC95\%: -0,37729; -0,29653), em comparação com 2008 (-0,16021; IC95\%: -0,23293; $0,08749)$. Os valores do IC e seus respectivos intervalos de confiança de $95 \%$ indicam que, no período de 1993 a 2008, houve redução estatisticamente significativa da concentração dos óbitos de menores de cinco anos entre aqueles cujas mães tinham baixa escolaridade.

Em 2008, o valor do IC para mortalidade na infância era mais elevado do que aquele para a mortalidade infantil. Isso indica que a concentração dos óbitos de menores de cinco anos entre crianças cujas mães têm menor escolaridade, em 2008, ainda era maior do que a concentração dos óbitos de menores de um ano, segundo a mesma característica. Essa concentração pode ser observada na Figura 3, que ilustra as curvas de concentração para mortalidade infantil e na infância, segundo a escolaridade materna, nos anos de 1993 e 2008.
A Figura 4 mostra a evolução do IC dos óbitos infantis e na infância, segundo a renda domiciliar per capita da mãe, no período de 1993 a 2008.

O IC para a mortalidade infantil segundo a renda domiciliar per capita apresentou maior variabilidade do que segundo a escolaridade materna. Em 1993, o IC foi -0,14307 (IC95\%: 0,23607; -0,05007), indicando concentração dos óbitos infantis entre as crianças cujas mães tinham menor renda domiciliar per capita. Em 2008, o IC foi 0,13521 (IC95\%: -0,01338; 0,28380). Apesar do valor pontual do IC ter sido positivo, o intervalo de confiança inclui o valor nulo. Assim, não é possível afirmar que houve inversão da direção da associação, mas sim que a concentração dos óbitos infantis entre as crianças cujas mães eram mais pobres deixou de existir.

O IC para a mortalidade na infância também apresentou grande variabilidade e comportamento semelhante ao IC para mortalidade infantil, segundo a renda domiciliar per capita. Em 1993, o IC foi -0,21287 (IC95\%: -0,25662; -0,16912), indicando concentração dos óbitos infantis entre as crianças cujas mães tinham menor renda domiciliar per capita. Ao longo do período, também se observa desconcentração. Em 2008, IC foi 0,03522 (IC95\%: -0,03928; 0,10972), indicando uma distribuição mais homogênea dos óbitos infantis segundo a renda domiciliar per capita da mãe. 
(a) Mortalidade infantil

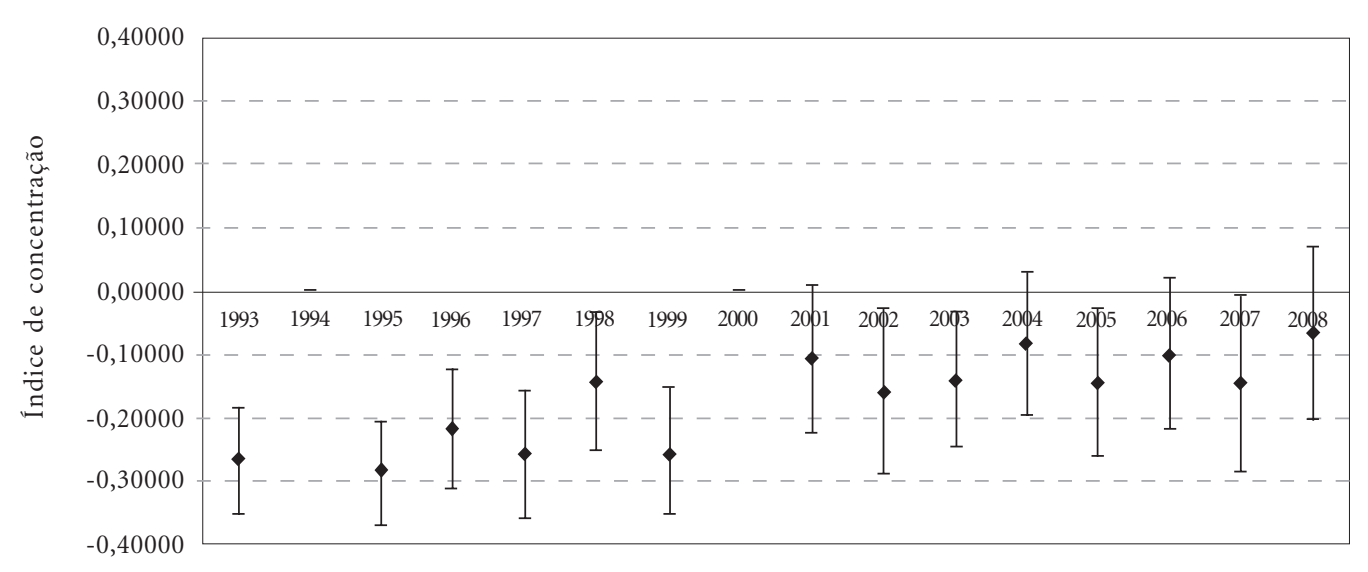

(b) Mortalidade na infância

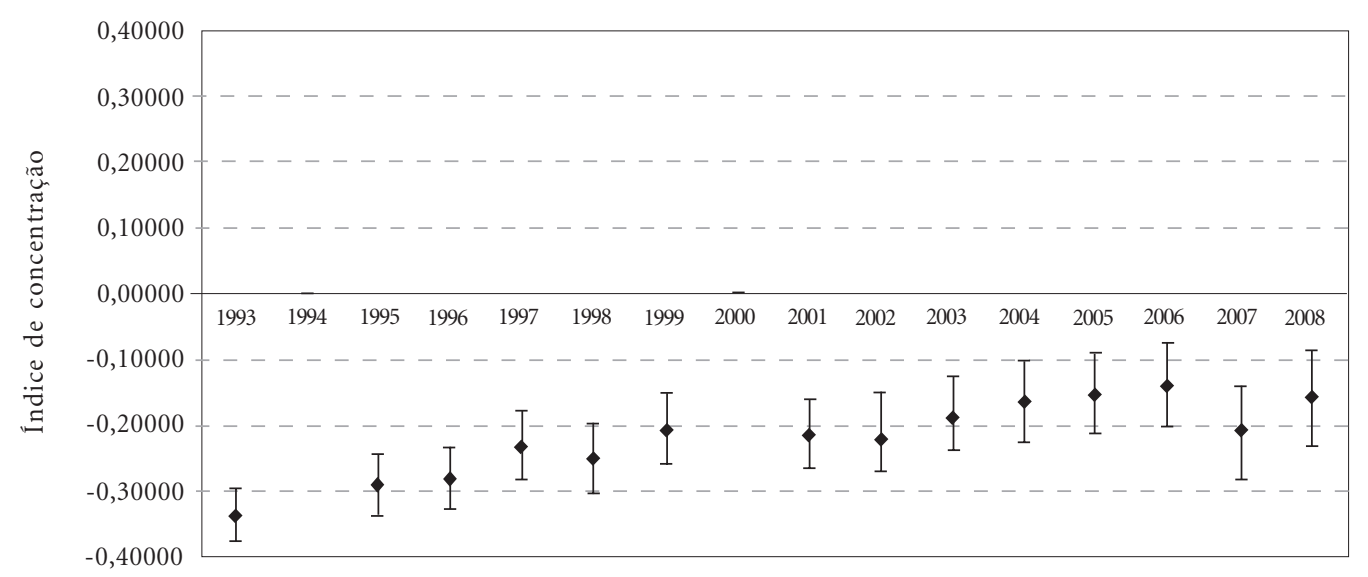

Figura 2. Índices de concentração e respectivos intervalos de confiança de $95 \%$ dos óbitos infantis (menores de um ano) (a) e na infância (menores de cinco anos) (b) segundo anos de estudo da mãe. Brasil, 1993 a $2008^{*}$.

* Elaboração dos autores, a partir dos microdados das Pesquisas Nacionais por Amostra de Domićlios (PNAD) 1993 a 2008. Nos anos de 1994 e 2000 não foi realizada PNAD.

\section{Discussão}

No presente estudo, analisou-se a evolução temporal da magnitude das desigualdades na mortalidade infantil e na infância, entre grupos definidos segundo a escolaridade materna e a renda domiciliar per capita no Brasil. No período de 1993 a 2008, observou-se redução das desigualdades na mortalidade infantil e na mortalidade na infância, segundo a escolaridade materna e a renda domiciliar per capita, analisada por meio dos IC e seus respectivos intervalos de confiança.
As medidas de diferença absoluta irão quase sempre levar a uma aparente redução nas desigualdades, pois os índices basais tendem a serem inicialmente mais baixos em termos absolutos entre os indivíduos com melhor nível socioeconômico. As medidas relativas levam em consideração os níveis basais e, portanto, permitem avaliar de maneira mais adequada se a desigualdade está aumentando ou diminuindo ${ }^{8}$. Entretanto, a renda média dos $20 \%$ mais ricos e $20 \%$ mais pobres pode variar no decorrer do tempo, assim como a proporção de mães nas categorias anali- 
(a) Mortalidade infantil

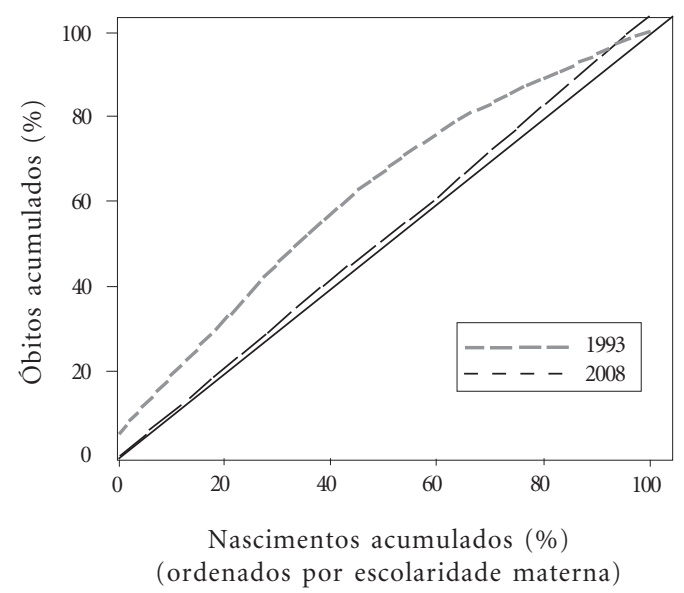

(b) Mortalidade na infância

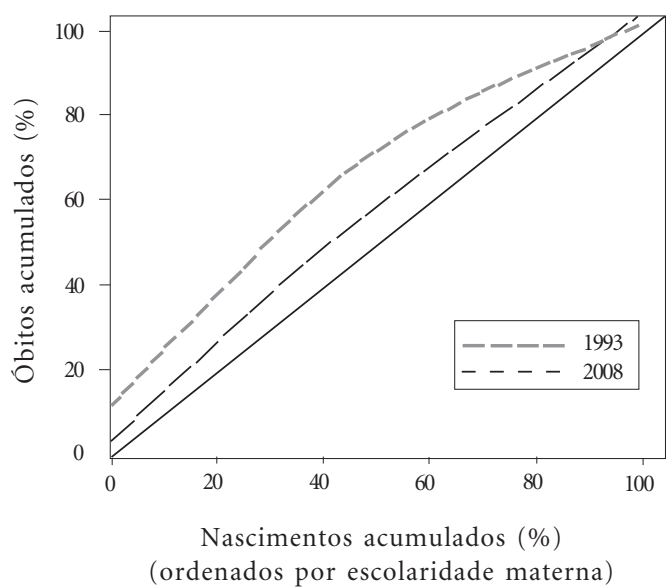

Figura 3. Curvas de concentração dos óbitos infantis (a) (menores de um ano) e na infância (b) (menores de cinco anos), segundo anos de estudo da mãe. Brasil, 1993 e $2008^{*}$

*Elaboração dos autores, a partir dos microdados das Pesquisas Nacionais por Amostra de Domicílios (PNAD) de 1993 e 2008.

sadas de escolaridade materna. Além disso, uma vez que a proporção de nascimentos nas diferentes categorias de renda e escolaridade pode variar ao longo do tempo, a comparação entre grupos extremos em diferentes anos poderia ser enviesada. Assim, mesmo a desigualdade relativa (risco relativo) não é a medida mais adequada para comparações temporais. Para isso, foi calculado o índice de concentração em saúde.

O IC tem como vantagens: (i) utiliza informações de todos os grupos sociais; (ii) incorpora dados sobre o tamanho dos grupos populacionais; (iii) não exige que os grupos sociais sejam ordenados; (iv) é válido para acompanhamento ao longo do tempo; e (v) permite a ilustração gráfica das tendências nas desigualdades em saúde 9 .

Os microdados das PNAD foram a fonte de dados para o presente estudo. A principal vantagem dos dados de estudos de base populacional sobre os sistemas de informação é que os primeiros permitem que as desigualdades em saúde sejam examinadas em relação a diversas dimensões do nível socioeconômico ${ }^{10}$.

O Sistema de Informação sobre Nascidos Vivos (Sinasc) foi implantado no Brasil em 1990. Seu documento base é a Declaração de Nascido Vivo (DN). A cobertura desse sistema é elevada e a qualidade da informação melhorou bastante nos últimos anos. Em 2000, havia falta de informação sobre a escolaridade materna em 5,5\% das DN, em 2007, essa proporção reduziu-se para $2,9 \%{ }^{11}$ Entretanto, a informação sobre a escolaridade materna nesse sistema é coletada de maneira categorizada (nenhuma; um a três anos; quatro a sete anos; oito a 11 anos; 12 e mais e ignorada). A ausência da informação sobre escolaridade materna medida em anos de estudo, como variável contínua, dificulta a realização de análises específicas de desigualdades, como o cálculo do IC. Além disso, a DN não coleta informações sobre renda domiciliar, nem familiar.

Apesar dessas vantagens dos estudos de base populacional sobre os sistemas de informação, a PNAD apresenta limitações importantes. Com relação a aspectos metodológicos da PNAD, é possível que o rendimento mensal domiciliar per capita esteja subestimado ${ }^{12}$. Isso pode ser consequência da imprecisão da informação, uma vez que esta foi obtida com base no relato do entrevistado. A desconsideração de informações relativas a patrimônio e outros rendimentos tende a minimizar as reais diferenças existentes entre os estratos mais pobres e mais ricos da população, já que subestima a renda destes últimos ${ }^{13,14}$. A influência de possíveis erros na estimativa da renda domiciliar per capita tenderia a subestimar os IC. 
(a) Mortalidade infantil

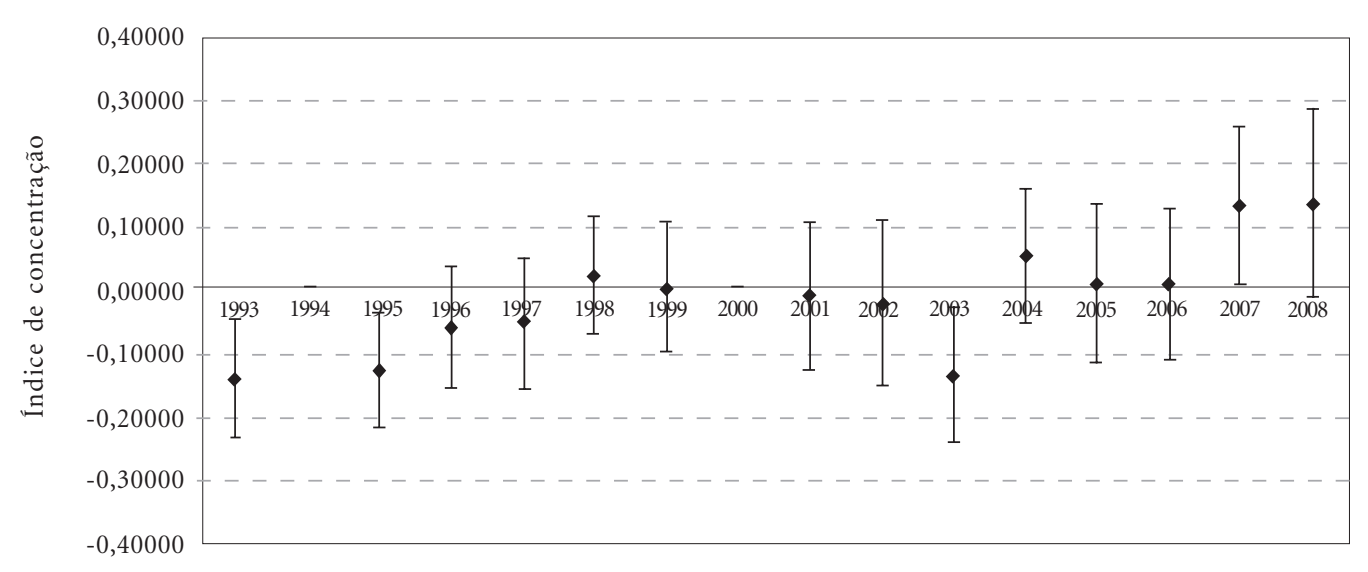

(b) Mortalidade na infância

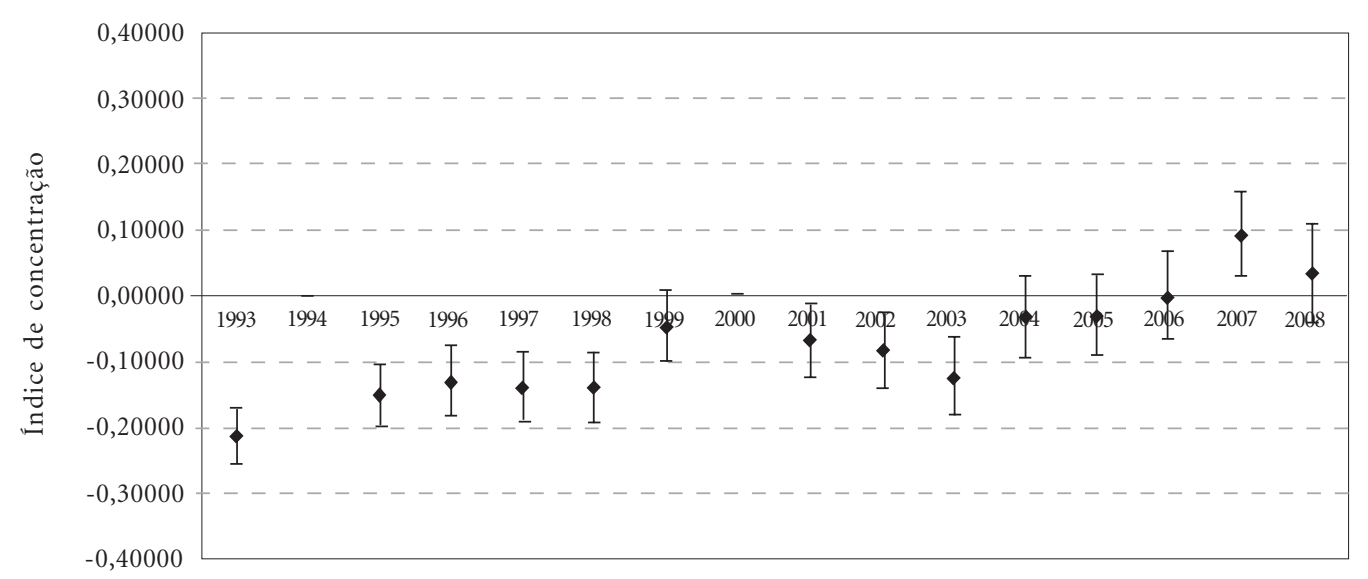

Figura 4. Índices de concentração e respectivos intervalos de confiança de 95\% dos óbitos infantis (menores de um ano) (a) e na infância (menores de cinco anos) (b) segundo renda domiciliar per capita. Brasil, 1993 a $2008^{*}$

"Elaboração dos autores, a partir dos microdados das Pesquisas Nacionais por Amostra de Domicílios (PNAD) 1993 a 2008. Nos anos de 1994 e 2000 não foi realizada PNAD.

Uma desvantagem na análise dos dados de inquéritos populacionais transversais, como a PNAD, é a possibilidade de ocorrência de viés de prevalência ou sobrevivência, uma vez que é esperado que os indivíduos com pior nível socioeconômico apresentem menor sobrevida. Isso pode resultar em problemas de seleção, pois as pessoas doentes e com menor renda tendem a estar sub-representadas entre os sobreviventes ${ }^{13}$. No presente estudo, os óbitos de crianças cujas mães também faleceram tendem a estar sub-representados. Considerando que os óbitos maternos são mais frequentes entre mulheres com menor renda e menor escolaridade, as medidas de desigualdade do presente estudo tendem a estar subestimadas.

Comparando-se as taxas de mortalidade infantil obtidas indiretamente a partir dos microdados das PNAD com aquelas calculadas diretamente a partir do número de nascidos vivos re- 
gistrados no SINASC e do número de óbitos de menores de um ano registrados no SIM, no período de 1993 a 2008, os valores não são muito diferentes. A taxa de mortalidade infantil obtida indiretamente, a partir dos dados da PNAD 2008, referente aos óbitos e nascimentos ocorridos em 2007, foi aproximadamente 11 óbitos por mil nascidos vivos, inferior àquela calculada diretamente a partir do SIM e do SINASC para o ano de 2007, de 15,7 óbitos por mil nascidos vivos. Para o ano de 2006, entretanto, essa diferença é menor. Enquanto a taxa de mortalidade infantil calculada diretamente a partir do SIM e SINASC foi 16,4 óbitos por mil nascidos vivos, aquela calculada a partir dos microdados da PNAD 2007 foi em torno de 14.

$O$ fato de, no presente estudo, terem sido considerados apenas os óbitos referentes ao último filho nascido vivo de cada mulher, também pode ter contribuído para a subestimação dos óbitos. A subestimação dos óbitos infantis, possivelmente, foi pequena. Para os óbitos na infância, entretanto, essa subestimação pode ter sido maior, uma vez que no período de cinco anos é possível que algumas mulheres entrevistadas tenham tido mais de um filho e que tenham ocorrido óbitos de outras crianças, que não eram o último filho nascido vivo dessas mulheres.

Ressalta-se que todos os dados analisados são provenientes de informações referidas pelos entrevistados e outra fonte potencial de erro relaciona-se ao uso de outro respondente para a entrevista. Diversos trabalhos discutem essa característica da $\mathrm{PNAD}^{13-16}$ No presente estudo, não se investigou possíveis diferenças nas proporções de respostas obtidas a partir de respondentes secundários. Dessa maneira, não se sabe como a utilização de outro respondente influenciou a mensuração das variáveis ${ }^{15}$.

Por outro lado, nos estudos em que o estado de saúde é referido pelo entrevistado, como é o caso da PNAD, a medição da mortalidade é mais robusta do que aquela da morbidade. Diversos autores discutiram a validade da informação referida sobre morbidades, uma vez que a doença pesquisada, a presença de comorbidades, bem como as características sociais e demográficas do respondente, podem afetar a qualidade dessa informação $0^{16-18}$. A informação sobre morbidade depende da percepção dos indivíduos, que pode ser diferente nos diferentes grupos socioeconômicos, o que é capaz, potencialmente, gerar vieses nas análises de desigualdades ${ }^{19}$. Os óbitos de crianças, por sua vez, são eventos incidentes e marcantes, portanto pouco sujeitos a viés de recordatório.
Outra limitação importante do presente estudo é que o tamanho da amostra não foi calculado para fornecer significância estatística às análises apresentadas. Amostras maiores poderiam fornecer maior poder e revelar diferenças que o presente estudo não foi capaz de demonstrar. Isso fica evidente quando se compara os dados do presente estudo sobre mortalidade infantil e na infância. Em virtude do maior número de observações para a mortalidade na infância, os intervalos de confiança para as estimativas das medidas de desigualdade desta mortalidade são mais estreitos do que aqueles para as estimativas da mortalidade infantil.

Além disso, a amostra da PNAD é estratificada segundo porte populacional, e não de acordo com características socioeconômicas. Dessa forma, com um pequeno número de observações, as amostras podem não ter sido representativas dos diferentes estratos sociais. O IC é uma medida de desigualdade amplamente empregada quando estão disponíveis microdados de pesquisas domiciliares com amostras complexas ${ }^{20}$. Na presente análise, foi incorporado o peso da pessoa no cálculo do IC.

Ainda, até 2003, a PNAD não cobria a zona rural da região Norte (exceto Tocantins). Foi apenas em 2004 que a PNAD passou a cobrir toda a zona rural brasileira. Essa modificação no plano amostral da PNAD pode afetar a comparabilidade temporal de diversos indicadores calculados a partir dos dados da PNAD, inclusive indicadores socioeconômicos ${ }^{21}$ e o IC calculado no presente estudo.

Apesar das limitações discutidas, os dados apresentados são úteis para analisar a evolução temporal da magnitude das desigualdades na mortalidade infantil e na infância, relacionadas à escolaridade materna e à renda domiciliar per capita no Brasil. Segundo essas variáveis, foi possível identificar uma tendência de redução das desigualdades, no nível individual, no período de 1993 a 2008, tanto para mortalidade infantil como na infância.

Existe um intenso debate na literatura a respeito das medidas empregadas para analisar as desigualdades em saúde $e^{2,22,23}$. Também existe um debate sobre quais variáveis independentes relacionadas ao nível socioeconômico devem ser empregadas no estudo das desigualdades em saúde $e^{2,24,25}$.

Tanto a renda quanto a escolaridade parecem estar associadas, de maneira robusta, com a situação de saúde. Os mecanismos causais envolvidos nessas associações também foram amplamente discutidos na literatura ${ }^{24-26}$. Apesar do gran- 
de volume de informação existente sobre a associação positiva entre renda e saúde, ainda não existe um consenso. É possível que essa associação seja o reflexo da relação não linear entre a renda e a saúde no nível individual, resultando em artefato estatístico. A saúde dos indivíduos que moram em vizinhanças pobres pode ser ruim não em decorrência de sua renda individual, mas em decorrência das condições de vida precárias em tal local, derivadas das desigualdades na sociedade como um todo. Todavia, claramente, essa hipótese não é suficientemente explicativa. Em países ricos, essa hipótese não se confirma, entretanto, em países pobres, um aumento na renda é positivamente associado com os níveis de saúde ${ }^{2}$. No presente estudo, optou-se por analisar duas dimensões do nível socioeconômico, a escolaridade materna e a renda domiciliar per capita, enfocando a medição das desigualdades, e não sua explicação.

A vantagem de se utilizar a escolaridade materna, em comparação com a renda domiciliar per capita é que a primeira é uma variável mais estável no tempo. No presente estudo, a informação da variável independente referiu-se ao momento da realização da entrevista, e não ao momento do óbito, nem ao do nascimento. Por isso, a análise das desigualdades na mortalidade infantil e na infância segundo a escolaridade, é mais robusta do que aquela conforme a renda. Além disso, é importante ressaltar que a renda domiciliar per capita empregada no presente estudo se refere às mães, e não às crianças. Considerando que a renda domiciliar per capita é calculada dividindo-se a soma das rendas individuais das pessoas residentes no domicílio pelo número de residentes, essa renda tende a se elevar em decorrência do óbito, uma vez que o denominador é reduzido. Assim, o IC para essa variável tende a ser subestimado.

Outros estudos investigaram as desigualdades socioeconômicas na mortalidade infantil e na infância. Wagstaff ${ }^{19}$ comparou essas desigualdades entre crianças em diferentes posições na distribuição de consumo, em nove países em desenvolvimento, entre eles o Brasil. Em muitos países existe um grande intervalo entre o quinto inferior na distribuição da riqueza e o resto da população. Apenas no Brasil, Nicarágua e Filipinas, a mortalidade declinou monotonicamente à medida que o consumo aumentava. O Brasil foi, disparado, o país com a distribuição mais desigual. Em todos os países, as desigualdades na mortalidade na infância foram mais pronunciadas do que na mortalidade infantil. Esse resultado foi consistente com aqueles do presente estudo.
Apesar dos dados das PNAD serem empregados para o cálculo das taxas de mortalidade infantil e na infância nos estados brasileiros que não têm cobertura adequada dos sistemas de informação em saúde, poucos estudos sobre desigualdades nestas mortalidades foram realizados a partir daquelas fontes de dados. Castro e Simões $^{27}$ analisaram as tendências espaçotemporais na mortalidade infantil no Brasil, estimada de maneira indireta, com base em dados das PNAD, no período de 1980 a 2005. Uma redução importante nas taxas de mortalidade infantil foi observada, todavia, os melhores resultados permaneceram concentrados nas regiões mais ricas do país. A desigualdade relativa entre as regiões mais ricas e mais pobres permaneceram inalteradas e, portanto, os óbitos infantis permaneceram desproporcionalmente concentrados nas regiões mais pobres.

Os achados do presente estudo são consistentes com os resultados de Monteiro et al. ${ }^{28}$ sobre as tendências na prevalência e distribuição social da desnutrição infantil no Brasil. Entre 1974 e 2008, foi documentando um forte declínio na prevalência de desnutrição, de $37,1 \%$ para $7,1 \%$. A prevalência reduziu-se de $59,0 \%$ para $11,2 \%$ no quinto mais pobre e de $12,1 \%$ para $3,3 \%$ no quinto mais rico. O declínio foi mais pronunciado nos últimos dez anos do período, quando os intervalos entre as famílias mais ricas e mais pobres com crianças menores de cinco anos também foram reduzidos em termos de poder de compra, acesso à educação, assistência à saúde e saneamento básico. Cabe ressaltar que a desnutrição é uma das causas de óbitos infantis e na infância.

Entretanto, estudo ecológico que investigou a evolução da desigualdade socioeconômica nas taxas de mortalidade neonatal e na infância estimadas indiretamente para os municípios brasileiros, revelou tendência divergente. Sousa et al..$^{29}$, em avaliação subnacional das tendências da desigualdade, no período de 1991 a 2000, verificaram que a redução nas taxas de mortalidade neonatal e na infância foi maior nos municípios mais ricos. Além disso, houve aumento da desigualdade relativa, medida como a razão entre as taxas dos $20 \%$ municípios mais pobres e $20 \%$ municípios mais ricos. A situação socioeconômica dos municípios foi classificada de acordo com a proporção da população abaixo da linha de pobreza.

Em outro estudo ecológico, Goldani et al. ${ }^{30}$ investigaram as tendências da mortalidade infantil de 1995 a 1999 de acordo com uma medida de base geográfica da escolaridade materna, em Porto Alegre. Observou-se declínio nos indica- 
dores de mortalidade, mais acentuado nas regiões mais pobres, de modo que as desigualdades nos componentes neonatal e pós-neonatal da mortalidade infantil foram reduzidas em relação à escolaridade materna.

Victora et al. ${ }^{31}$ discutiram questões de equidade em relação à saúde e à mortalidade infantil. Segundo os autores, países e pessoas pobres têm múltiplas privações. Essas privações se relacionam a níveis elevados de exposição a doenças, níveis baixos de resistência, busca inadequada por cuidados de saúde e probabilidades mais baixas de receber tratamento efetivo e oportuno. Para minimizar as desigualdades, são discutidas as vantagens e desvantagens de intervenções focalizadas e universais. Os autores concluem que a equidade deve ser uma prioridade no planejamento das intervenções e estratégias voltadas à saúde infantil.

As grandes melhorias nos indicadores de saúde infantil observados no Brasil nas últimas décadas refletem tendências positivas e equitativas nos determinantes proximais e distais da saúde infantil que resultam do crescimento econômico em geral e de políticas públicas orientadas para a equidade $^{28}$. Especificamente, para a mortalidade infantil, a Estratégia de Saúde da Família teve um papel importante. Pesquisa realizada pelo Ministério da Saúde, em parceria com pesquisadores da Universidade de Nova York, buscando avaliar o impacto dessa Estratégia nas taxas de mortalidade infantil em nível estadual, realizou análise ecológica longitudinal de 1990 a 2002, com dados de painel de fontes secundárias de todos os 27 estados do Brasil. Nesses 13 anos, a taxa de mortalidade infantil declinou de 49,7 para 28,9 por mil nascidos vivos. Durante o mesmo período, a cobertura do PSF aumentou de zero para 36\% da população. Um aumento de $10 \%$ na cobertura do PSF foi associado a uma redução de 4,5\% na taxa de mortalidade infantil, controlando para todos os outros determinantes de saúde ${ }^{32}$.

A medição das desigualdades em saúde também é importante para o acompanhamento da situação de saúde da população, uma vez que uma alteração no valor de um indicador pode derivar de diferentes alterações em sua distribuição na população. Assim, uma redução na mortalidade infantil e na infância não significa, necessariamente, uma redução na desigualdade. Por exemplo, essa redução pode ocorrer em consequência de uma grande melhoria entre crianças de melhor condição socioeconômica, porém, às custas de uma deterioração nas crianças com pior condição socioeconômica. Em outro cenário, a redução na mortalidade infantil e na infância poderia ocorrer com a manutenção das desigualdades. O melhor cenário, que combina melhoria no indicador e redução das desigualdades, foi identificado no presente estudo. Pesquisas futuras, com dados provenientes de amostras maiores ou dados dos registros vitais baseados nos sistemas de informação em saúde, bem como utilizando técnicas indiretas para estimar a mortalidade, poderão confirmar essa tendência e demonstrar se a mesma será mantida.

\section{Colaboradores}

LP Garcia e LR Santana foram responsáveis pelo delineamento do estudo, análise e interpretação dos dados e redação do artigo. 


\section{Referências}

1. Instituto de Pesquisa Econômica Aplicada. Objetivos de desenvolvimento do milênio: relatório nacional de acompanhamento. Brasília: Ipea; 2010.

2. Masseria C, Hernández-Quevedo C, Allin S. Health inequality: what does it mean and how can we measure it? Expert Rev Pharmacoeconomics Outcomes Res. 2010; 10(2):177-186.

3. França E, Lansky S. Mortalidade infantil neonatal no Brasil: situação, tendências e perspectivas. In: Demografia e saúde: contribuição para a análise de situação e tendências. Série G. Estatística e Informação em Saúde Série Informe de Situação e Tendências. Brasília: Organização Pan Americana da Saúde; 2009: p. 83-112.

4. World Health Organization. World health statistics 2010. WHO; 2010.

5. Konings P, Harper S, Lynch J, Hosseinpoor AR, Berkvens D, Lorant V, Geckova A, Speybroeck N. Analysis of socioeconomic health inequalities using the concentration index. Int J Public Health 2009; 55(1):71-74.

6. O’Donnell O, The World Bank. The concentration index. In: Analyzing health equity using household survey data: a guide to techniques and their implementation. Washington D.C: World Bank; 2008.

7. Kakwani N, Wagstaff A, van Doorslaer E. Socioeconomic inequalities in health: Measurement, computation, and statistical inference. Journal of Econometrics. 1997; 77(1):87-103.

8. Victora C, Vaughan J, Barros F, Silva A, Tomasi E. Explaining trends in inequities: evidence from Brazilian child health studies. The Lancet 2000; 356(9235):1093-1098.

9. Harper S, Lynch J, Meersman SC, Breen N, Davis WW, Reichman ME. An Overview of Methods for Monitoring Social Disparities in Cancer with an Example Using Trends in Lung Cancer Incidence by Area-Socioeconomic Position and Race-Ethnicity, 1992-2004. American Journal of Epidemiology. 2008; 167(8):889-899.

10. O'Donnell, Owen. Measuring health inequalities in Europe: Methodological issues in the analysis of survey data. Eurohealth. 2009; 15(3):10-14.

11. Nogales AM, Porto D. Saúde Reprodutiva: como nascem os brasileiros. In: Ministério da Saúde (MS). Saúde Brasil 2009: uma análise da situação de saúde e da agenda nacional e internacional de prioridades em saúde. Brasília, MS; 2010. p.21-42.

12. Hoffmann R. A subdeclaração dos rendimentos. São Paulo em Perspectiva 1988; 2(1):50-54.

13. Lima-Costa MF, Barreto S, Giatti L, Uchôa E. Desigualdade social e saúde entre idosos brasileiros: um estudo baseado na Pesquisa Nacional por Amostra de Domicílios. Cad. Saúde Pública. 2003;19(3). [acessado 2010 ago 20]. Disponível em: http:// www.scielo.br/scielo.php?script=sci_arttext\&pid= S0102-311X2003000300007\&lng=pt\&nrm=iso\&tlng $=$ pt.
14. Lima-Costa MF, Barreto S, Giatti L. A situação socioeconômica afeta igualmente a saúde de idosos e adultos mais jovens no Brasil? Um estudo utilizando dados da Pesquisa Nacional por Amostras de Domicílios \&shy; PNAD/98. Cien Saude Colet 2002; 7(4). [acessado 2010 ago 20]. Disponível em: http:/ /www.scielo.br/scielo.php?script=sci_arttext\&pid $=$ S1413-81232002000400015\&lng $=$ pt $\& n r m=i s o \&$ tlng $=$ pt.

15. Jardim R, Barreto SM, Gonçalves LG. Confiabilidade do informante secundário em inquéritos de saúde. R Bras Est Pop. 2009; 26(1):141-144.

16. Barros MBDA, César CLG, Carandina L, Torre GD. Desigualdades sociais na prevalência de doenças crônicas no Brasil, PNAD-2003. Cienc Saude Colet 2006; 11(4):911-926.

17. Okura Y, Urban L, Mahoney D, Jacobsen S, Rodeheffer R. Agreement between self-report questionnaires and medical record data was substantial for diabetes, hypertension, myocardial infarction and stroke but not for heart failure. J Clin Epidemiol. 2004; 57(10):1096-1103.

18. Simpson CF, Boyd CM, Carlson MC, Griswold ME, Guralnik JM, Fried LP. Agreement Between SelfReport of Disease Diagnoses and Medical Record Validation in Disabled Older Women: Factors That Modify Agreement. J Am. Geriatr Soc 2004; 52(1):123127.

19. Wagstaff A. Socioeconomic inequalities in child mortality: comparisons across nine developing countries. Bull World Health Organ 2000; 78(1):1929.

20. O’Donnell O, World Bank. Analyzing health equity using household survey data : a guide to techniques and their implementation. Washington D.C.: World Bank; 2008.

21. Rede Interagencial de Informações para a Saúde; Pan American Health Organization. Indicadores básicos para a saúde no Brasil: conceitos e aplicações. $2^{\text {a }}$ ed. Brasília: Organização Pan Americana da Saúde; 2008.

22. Deaton A. Health, Inequality, and Economic Development. J Econ Lit 2003; 41(1): 113-158.

23. Houweling T, Kunst A, Mackenbach J. Measuring health inequality among children in developing countries: does the choice of the indicator of economic status matter? Int J Equity Health 2003; 2(1):8.

24. Lynch J, Smith GD, Harper S, Hillemeier M, Ross N, Kaplan GA, Wolfson M. Is income inequality a determinant of population health? Part 1. A systematic review. Milbank Q. 2004; 82(1):5-99.

25. Wilkinson R, Pickett K. Income inequality and population health: A review and explanation of the evidence. Soc Sci Med 2006; 62(7):1768-1784.

Kawachi I, Adler NE, Dow WH. Money, schooling,

26. and health: Mechanisms and causal evidence. Annals of the New York Academy of Sciences. 2010; 1186(1):56-68. 
27. Castro M, Simões C. Spatio-temporal trends of infant mortality in Brazil. In: XXVI IUSSP International Population Conference. Marrakech; 2009. [acessado 2010 ago 20]. Disponível em: http://iussp 2009.princeton.edu/download.aspx?submissionId $=92270$

28. Monteiro CA, Benicio MHD, Conde WL, Konno S, Lovadino AL, Barros AJ, Victora CG. Narrowing socioeconomic inequality in child stunting: the Brazilian experience, 1974-2007. Bull. World Health Organ 2010; 88(4):305-311.

29. Sousa A, Hill K, Dal Poz M. Sub-national assessment of inequality trends in neonatal and child mortality in Brazil. International Journal for Equity in Health 2010; 9(1):21.

30. Goldani MZ, Benatti R, Silva AAMD, Bettiol H, Correa JCW, Tietzmann M, Barbieri MA. Narrowing inequalities in infant mortality in Southern Brazil. Rev Saude Publica 2002; 36(4):478-483. [acessado 2010 ago 20]. Disponível em: http://www.scielo. br/scielo.php?script $=$ sci_arttext $\&$ pid $=$ S0034$89102002000400014 \& \operatorname{lng}=$ en \&nrm=iso\&tlng=en.

31. Victora C, Wagstaff A, Schellenberg J, Gwatkin D, Claeson M, Habicht JP. Applying an equity lens to child health and mortality: more of the same is not enough. The Lancet 2003; 362(9379):233-241.

32. Macinko J. Evaluation of the impact of the Family Health Program on infant mortality in Brazil, 19902002. Journal of Epidemiology \& Community Health 2006; 60(1):13-19.

Artigo apresentado em 23/09/2010

Aprovado em 04/04/2011

Versão Final apresentada em 05/05/2011 